\title{
GP \\ RESEARCH \\ 4.5-Mb YAC STS Contig at 50-kb Resolution, Spanning Xq25 Deletions in Two Patients with Lymphoproliferative Syndrome
}

\author{
Giovanni Porta, ${ }^{1,2}$ Sandra MacMillan, ${ }^{1}$ Ramaiah Nagaraja, ${ }^{1}$ \\ Steven Mumm, ${ }^{1}$ lleana Zucchi, ${ }^{1,3}$ Giuseppe Pilia, ${ }^{1}$ Shirong Maio, ${ }^{1}$ \\ Terence Featherstone, ${ }^{1}$ and David Schlessinger ${ }^{1,4}$
}

\author{
'Department of Molecular Microbiology and Center for Genetics in Medicine, Washington University \\ School of Medicine, St. Louis, Missouri 63110; ${ }^{2}$ Department of Human Genetics, II Facolta di Medicina, \\ Pavia, Italy; ${ }^{3}$ Istituto Technologie Biomedicale Avanziata, 20131 Milano, Italy
}

Sequence-tagged site (STS) content mapping in yeast artificial chromosomes (YACs) was used to cover the region deleted in two patients affected with X-linked lymphoproliferative disorder. The order of markers includes, centromere to telomere, DXS8009-DXS1206-DXS8078-DXS8044-DXS982-DXS6811-DXS8093AFM240xb10-DXS75-DXS737-DXS100-DXS6-DXS1046-DXS8038. The order of six major markers is confirmed by fluorescent in situ hybridization, and all the markers assigned by linkage mapping fall within a 1.6-cM interval. The contig comprises 90 clones containing 89 STSs, yielding a resolution of $50 \mathrm{~kb}$; DNA in a gap just telomeric to DXS8044 has not been found in $>20$ equivalents of YACs or bacterial clones. The two deletions were found to have centromeric breakpoints that lie close to DXS1206 and may be identical; the telomeric breakpoints are $\sim 150 \mathrm{~kb}$ apart, one falling between DXS737 and DXS100, the other between DXS100 and DXS1046. Several STSs near the breakpoints show weak amplification from more than one site; one gives products from three groups of YACs, and lie, respectively, within $50 \mathrm{~kb}$ of the centromeric and the two telomeric deletion borders. Such partially duplicated segments of DNA are candidates for involvement in the formation of the deletions.

[A hyperlinked version of Table 1 is available at http://www.cshl.org/gr.]

Among the group of X-linked immunodeficiencies, lymphoproliferative disorder (LYP; MIM308240) is unique in causing a specific defect in the $T$ cell-mediated response to Epstein-Barr virus (Bare et al. 1974; Purtilo et al. 1977). As a result, patients progress to disease that varies from agammaglobulinemia to the development of lymphomas. The pathogenesis of the disease involves massive tissue destruction resulting from infiltration of activated $\mathrm{T}$ cells and macrophages. Extranodal lymphoma has been observed in many patients, and has been linked to a complex interaction with the mechanism of apoptosis. In fact, in a mouse model for one form of lymphoproliferative disease, the cell surface antigen fas, known to be involved in lymphocyte apoptosis, is defective in both $\mathrm{T}$ and $\mathrm{B}$ cells infected with Epstein-Barr virus (Ogasawara et al. 1993).

${ }^{4}$ Corresponding author.

E-MAIL davids@sequencer.wustl.edu; FAX 314-362-3203.
The severe cytotoxic T-cell response in LYP has been ascribed by different groups to polyclonally activated alloreactive cytotoxic T cells or to unregulated autoimmune killer and natural killer cell activity against both EBV-infected and -uninfected cells (Sullivan and Woda 1989). These EBVprovoked cells show cytotoxicity not only to EBVinfected B cells, but also to fibroblast and hepatocyte target cells, so that some host component is also attacked; but recent reviews (Sullivan and Woda 1989; Conley 1992) have pointed out that traditional approaches to pathophysiology have left unclear the nature of the immunological crippling by LYP, the basis of hepatic damage and liver failure, and the reason for variable responses. In contrast, direct study of the gene could reveal the causative factor and the nature and extent of relevant interactions (Seemayer et al. 1995). 
PORTA ET AL.

Positional cloning to find the disease gene has seemed more feasible since a number of linkage mapping studies and analysis of a large deletion restricted LYP to Xq25 (Wyandt et al. 1989). The candidate region was further refined when two affected boys from different families showed smaller deletions (Skare et al. 1993), detected by the absence of Xq25-specific probes. Wu et al. (1993) ordered several of the probes and suggested from in situ hybridization that the deletions were $\sim 2 \mathrm{Mb}$ in extent.

We have constructed a yeast artificial chromosome (YAC) sequence-tagged site (STS) contig that spans the two deletions reported (Wu et al. 1993). The STS content mapping results (1) reorder DXS6 and DXS100 and provide long-range cloned coverage of the region, which proves to be $\sim 3 \mathrm{Mb}$; (2) place and order a number of additional markers that detect polymorphism and could narrow the localization of the disease gene; and (3) hint at a possible origin for the deletion formation.

\section{RESULTS}

\section{Construction of the Map in Relation to Deletion Borders}

The current map of the region is shown in Figure 1. Mapping began with the recovery of cognate YACs for a set of probes (DXS100, DXS739, DXS75, DXS6, and DXS982) that had been shown to lie in or near the LYP locus in previous studies (Bare et al. 1974; Wu et al. 1993; note that other probes that had been shown to be linked to LYP, including DXS42, were excluded from the deletion span and were not specifically studied further to develop this contig). Some overlaps among the initial set of YACs, determined by STS content, provided coverage of about one-third the entire region. Mapping then continued by repeated cycles of end-walking (Kere et al. 1992), with the reiterative synthesis of new STSs from insert ends of YACs at the borders of nascent contigs. The STSs were also supplemented with new polymorphic markers and some additional probes in the region as they became available from the community or from screening efforts in the Washington University Genome Center. In every case the STSs were tested against the two deletion DNAs to verify that mapping was continuing in the target zone, and to permit the recognition of the borders of the deletion regions when they were crossed by YACs.

Figure 1 contains 85 YACs, selected for quality and coverage from a total of 140 recovered from six YAC libraries; five PACs for two loci are also in- cluded. The map is formatted with 89 STSs (Table 1) across $\sim 4.5 \mathrm{Mb}$ of DNA. This brings the resolution of ordered, relatively well-spaced markers to $\sim 50 \mathrm{~kb}$. We note that the STSs include seven dinucleotide repeat markers on the Centre d'Etude du Polymorphisme Humain (CEPH) Genethon linkage map and a tetranucleotide repeat marker from the Cooperative Human Linkage Center (CHLC).

The internal consistency of STS content in the

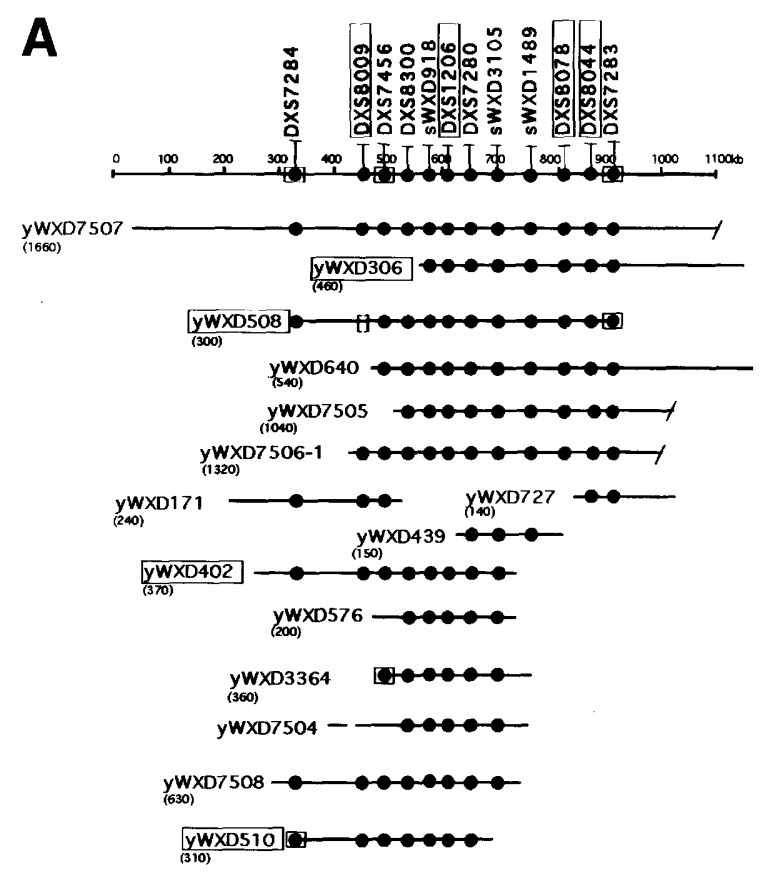

Figure 1 The map was inferred by the SEGMAP program (see Methods). A gap intervenes between the shorter $1-\mathrm{Mb}$ centromeric portion $(A)$ and the $4-\mathrm{Mb}$ telomeric portion $(B)$, with the two segments drawn in slightly different formats for clarity (see text). In a few instances, an STS was apparently deleted in a YAC, and is indicated by a vertical bracket. YACs are drawn proportional to their DNA content. STS content is indicated by a filled circle, or a square that indicates the origin of an STS from a YAC insert end. The single heavy bar under the distance scale shows a point at which only a single clone bridges two regions of the contig. Dashed YACs are larger in size than their STS content indicates, suggesting possible chimerism. The size of each YAC (and the calculated size for dashed YACs in kb) are given in parentheses at the left of each represented clone. STSs are indicated by DXS nos. where available, and by sWXD accession nos. for those still being processed at the Genome Database; YACs are indicated by YWXD nos., and PACs by pWXD nos. (see Methods and Table 1). Boxed YAC names indicated clones placed in the region by FISH. Boxed STSs (top scale) are Genethon markers that detect polymorphism. 


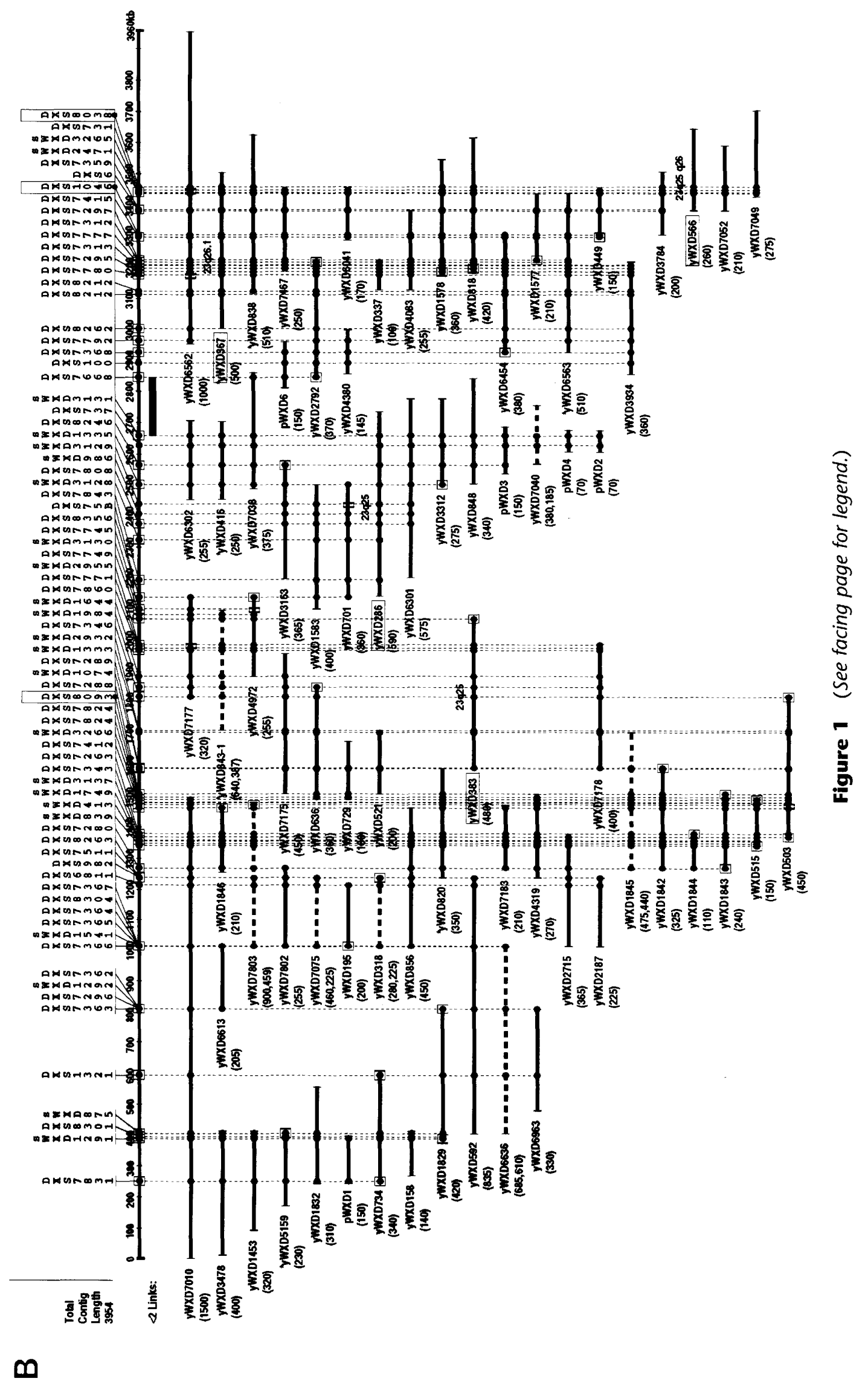




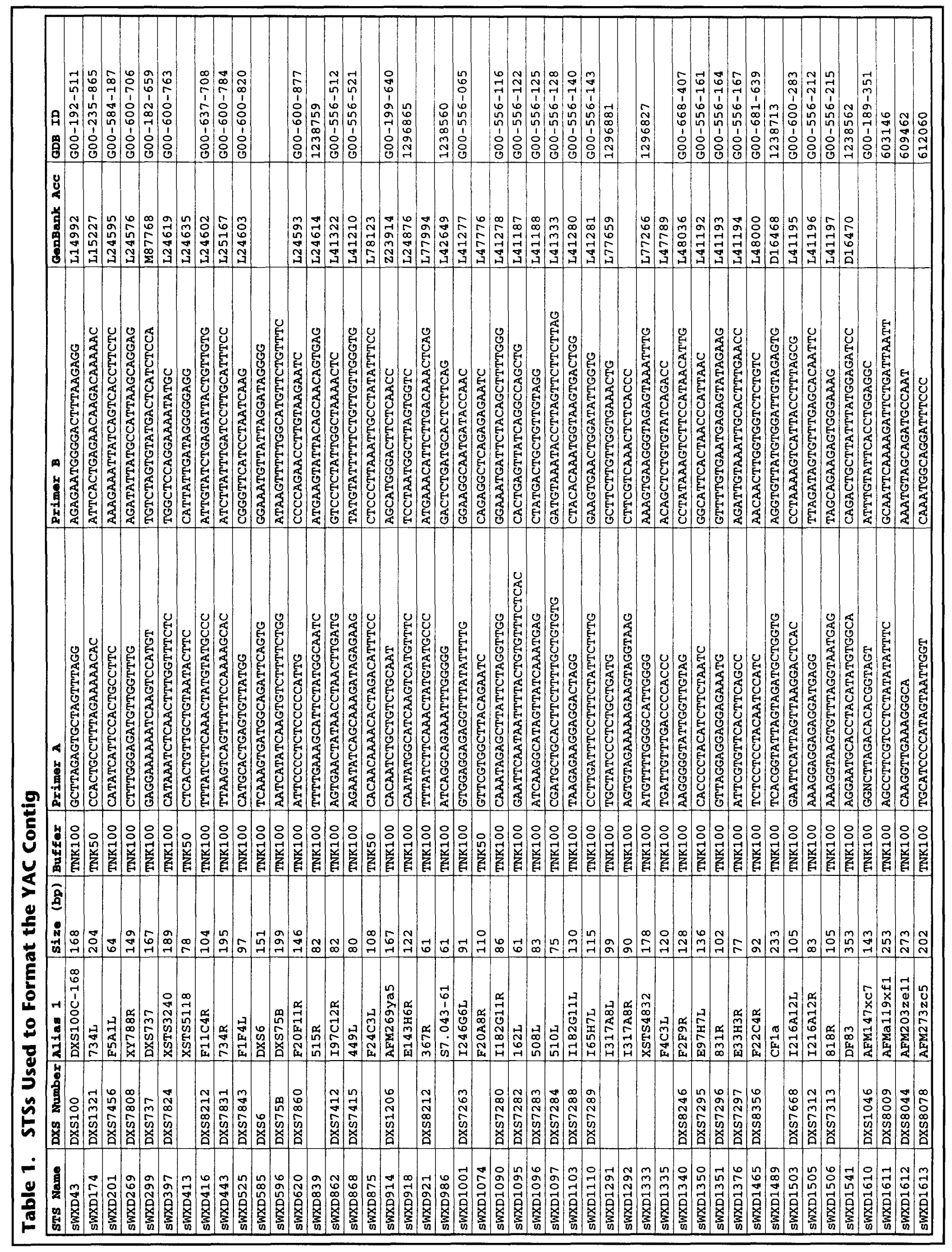


Downloaded from genome.cshlp.org on April 25, 2023 - Published by Cold Spring Harbor Laboratory Press

YAC CONIIG SPANNING LYP DELETIONS IN Xo25

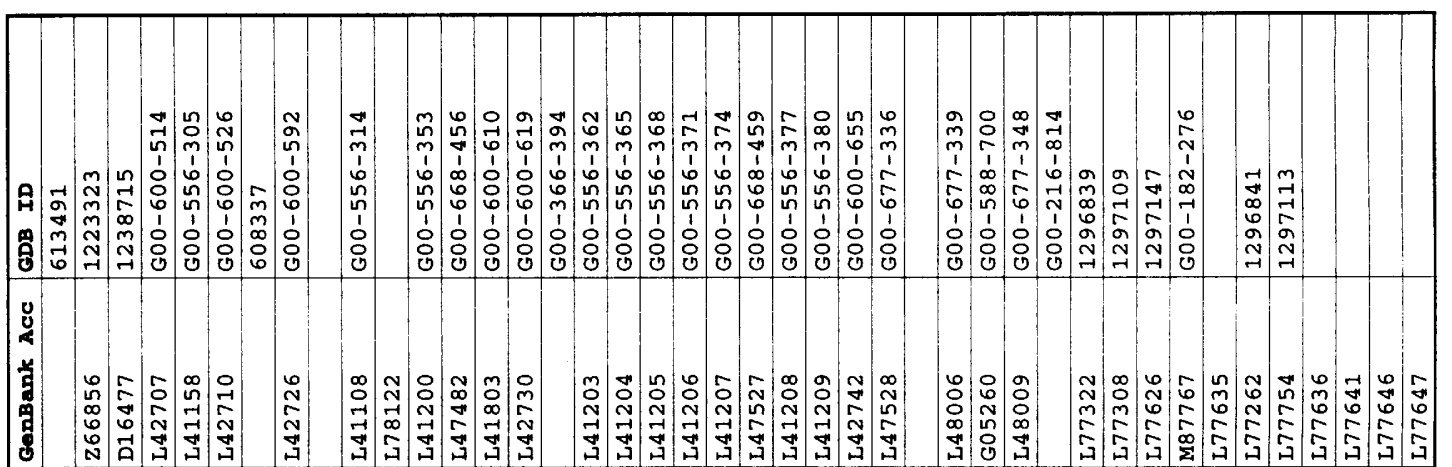

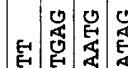

u

U⿺辶寸

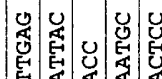

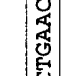

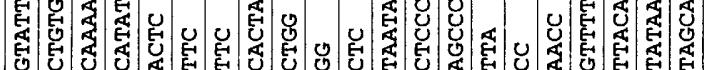

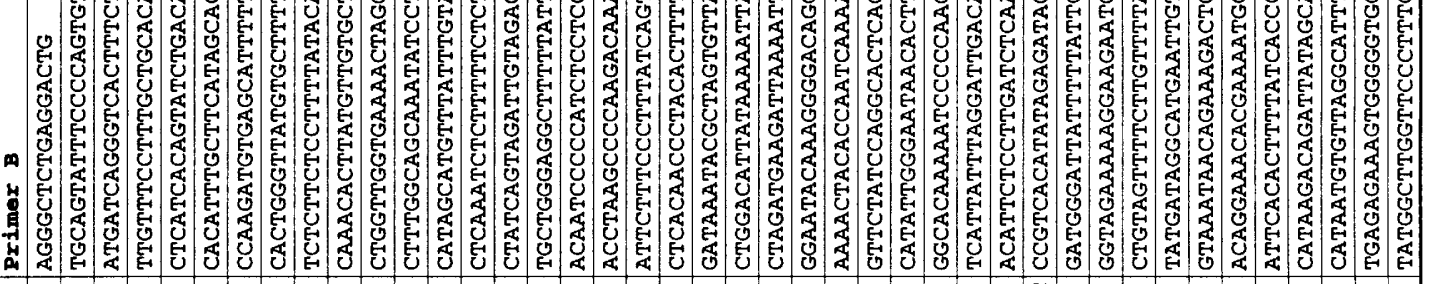

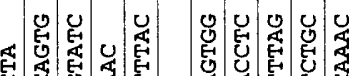

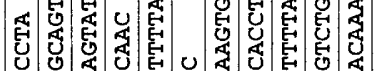

:

$\mid$

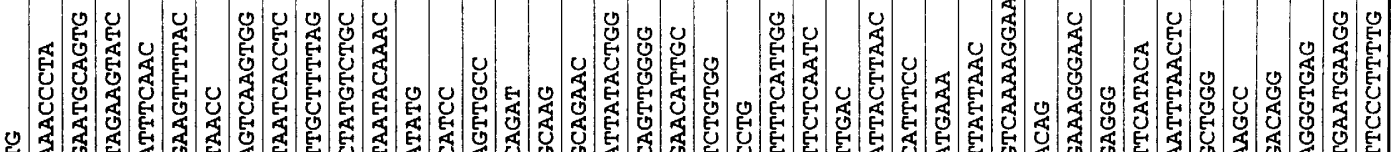

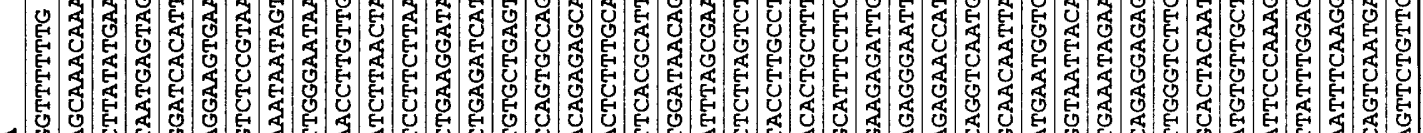

要 4 J

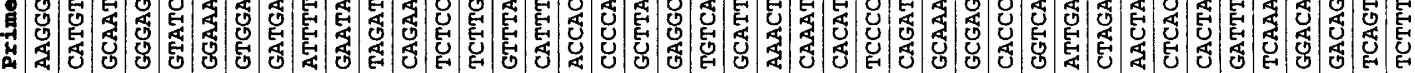

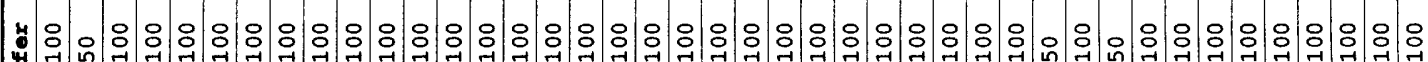

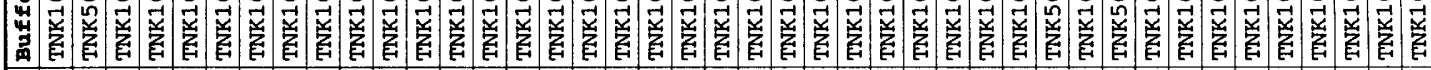
毵

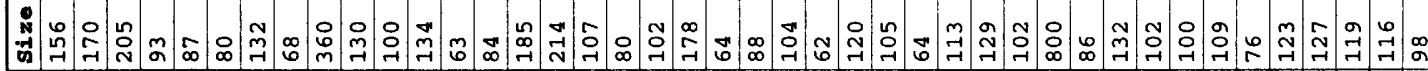

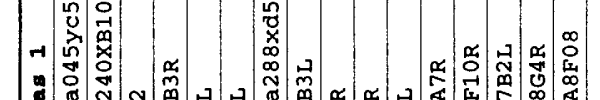

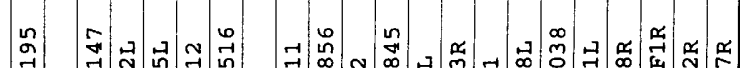

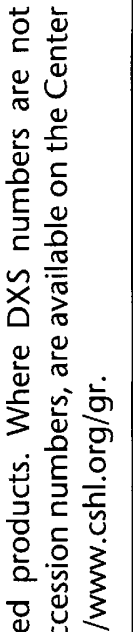

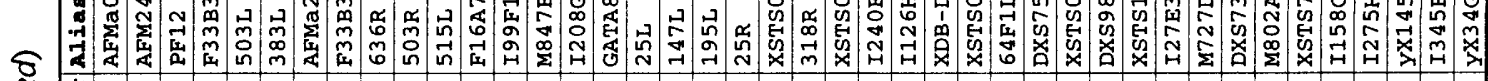

总 宜

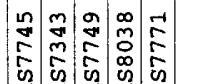

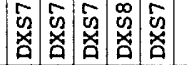

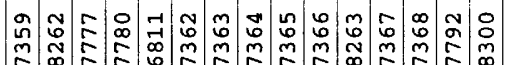

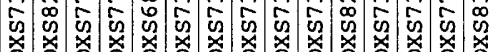

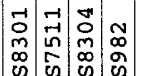

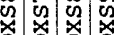

$\stackrel{-1}{m}$

.

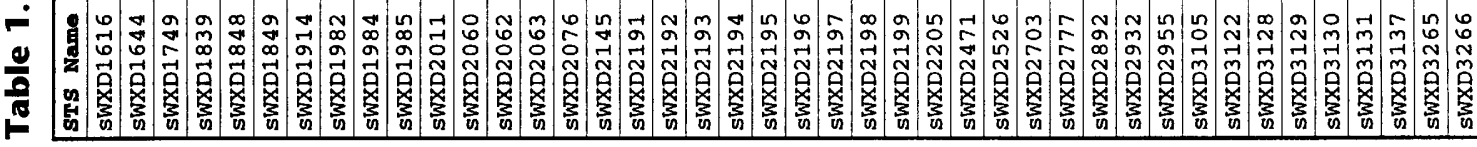

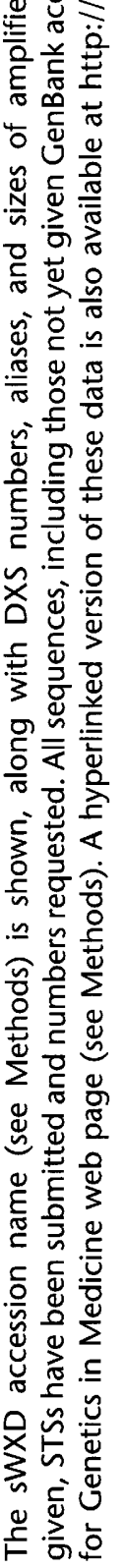


YACs was one indication of the validity of the map. A second came from the self-consistent patterns of Alu- and L1-containing TaqI fragments generated from YACs spanning about half the interval covered by the contig; as in the case of Xq27 (Zucchi et al. 1996), there were no discrepancies observed in the profile obtained from YACs from several different collections (data not shown).

In another approach to verification of marker order, inter-Alu products from YACs containing each of a set of markers were differentially labeled and ordered by fluorescent in situ hybridization (FISH). An example is given in Figure 2. Along a chromosome that is incompletely isolated but oriented by its banding pattern, clones containing the markers DXS1206, DXS982, and DXS100 lie in that order from centromere to telomere. The results are in agreement both with the contig map of Figure 1 and with pairwise hybridizations of the markers (not shown).

Still another set of results that are strongly consistent with the order of markers as shown came when each STS was tested against DNA from each of the two deletion patients $739(3.5 \mathrm{Mb})$ and $503(3$ $\mathrm{Mb})$, and its position compared with the inferred order of Figure 1. Sharp borders were seen between the STSs included and excluded from each deletion, with complete consistency as indicated in the summary of Figure 3. Each breakpoint was localized within a distance of $\sim 100 \mathrm{~kb}$. The centromeric breakpoints for both deletions fell between STSs DXS7284 (sWXD1097) and DXS8009 (sWXD1611), and the two telomeric breakpoints fell at two distinct locations $\sim 200 \mathrm{~kb}$ apart--between STSs sWXD3131 and DXS7668 (sWXD1503), and DXS8262 (sWXD2062) and DXS8212 (sWXD416), respectively.

For one location, the right end of yWXD734, no DNA extending appreciably further in the centromeric direction was recovered in 10 genomic equivalents of YACs or 10 equivalents of cosmids and bacterial artificial chromosomes (see Discussion). The contigs centromeric and telomeric of the remaining gap (Fig. 1) are aligned and oriented by links to adjoining contigs and by additional cytogenetic and genetic analyses.

The resolution and clone depth of the map was sufficient to resolve several apparent discrepancies in the ensemble of the data. For example, a megaYAC, 802A8, contained a large number of STSs from the region around DXS1206, but an STS made from an end of the YAC insert mapped several megabases further toward the telomere, near DXS75. Because none of the STSs in the intermedi- ate region established by the YAC STS contig were found in the megaYAC, it was inferred to contain a large internal deletion, and has not been shown in the map of Figure 1.

Sequences of YAC insert ends in the region were generally low in GC content. The percent GC for a random selection of 26 is listed in Table 2; most of them were too low in GC content to afford STS development. The overall level of GC (34\%) corresponds to the very lowest GC content DNA in the genome, indicative of a low content of genes (see Discussion).

\section{Duplicated STSs}

From the start of mapping in this region, some hybridization probes and STSs showed weak signals with clones from apparently different locations. Scoring of STSs could be made less ambiguous by using more stringent conditions for the PCR amplification step, but some ambiguities remained. An extreme example of apparently regionally duplicated sequences was seen with STSs from the ends of YAC yWXD162. Clone yWXD162 yields amplification products for four STSs that fall in a single set of YACs (sWXD918, 914, 1095, and 2205 in Fig. 1). STSs from both insert ends also amplified products only from YACs in Xq25, but at more than one site.

$162 \mathrm{R}$, an STS from the right insert end of the YAC, gave amplification signals from a second location more centromeric to the contig shown here (data not shown), suggesting that $162 \mathrm{R}$ contains a partially duplicated sequence. More extensive indications of duplication were seen with the STS from the other end of the YAC (sWXD1095, 162L). It amplified a product of the appropriate size from YACs at three different locations. One position coincided with the location of the four internal STSs in yWXD162, near DXS1206. The other two included some YACs just centromeric and another group just telomeric to DXS100. PCR assays with two clones from each region are shown in Figure 4. [Consistently, YACs from two of the locations yielded a second, less intense amplification product of somewhat smaller size. The secondary product was never seen with total human DNA, and may represent a truncated STS (R. Nagaraja and S. MacMillan, unpubl.).] Suggestively, these three positions fall, respectively, near the telomeric breakpoints of the larger and smaller deletions and near the centromeric breakpoint(s) of both deletions.

\section{DISCUSSION}

In addition to the self-consistency of STS content in 


\section{YAC CONTIG SPANNING LYP DELETIONS IN Xo25}
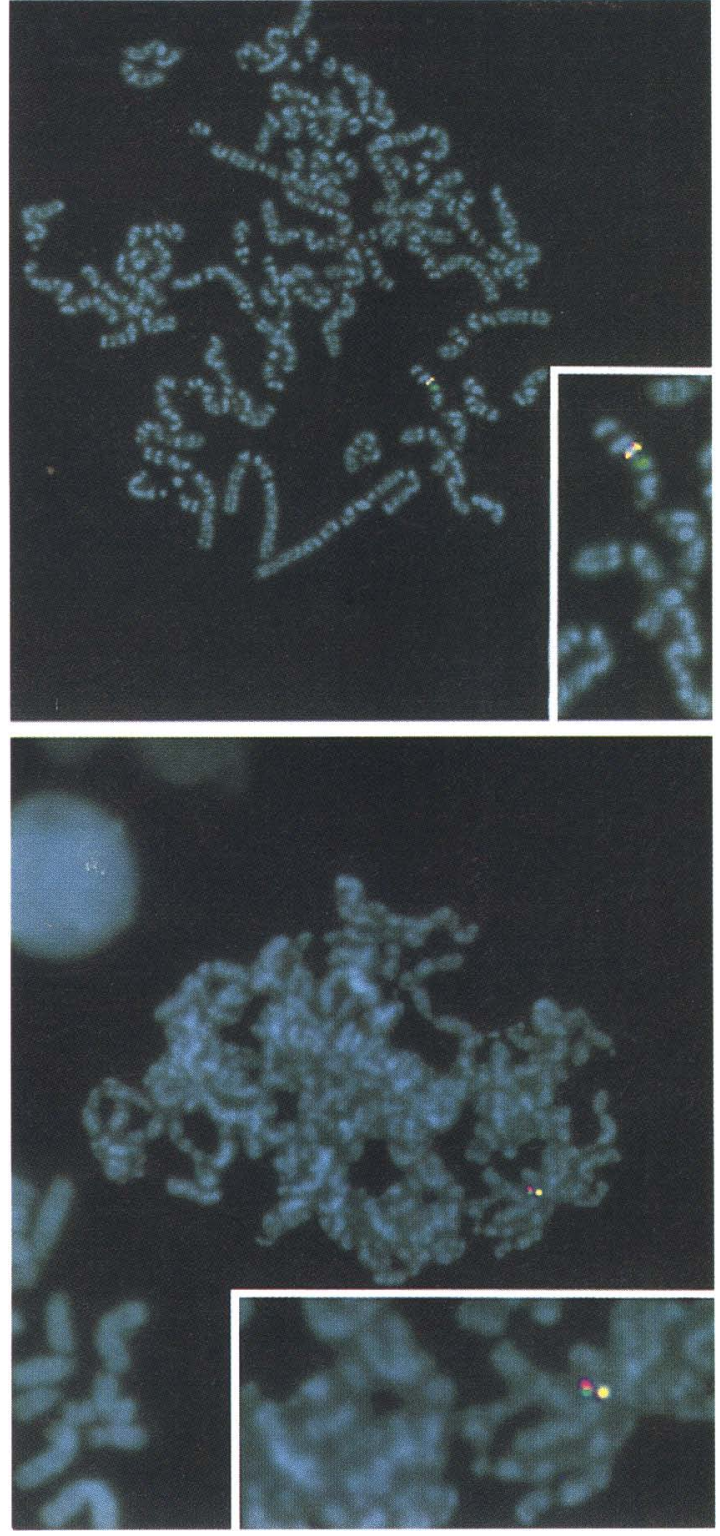

the YACs, the contig as shown in Figure 1 is verified by auxiliary fingerprinting assays, and by FISH studies of YACs and cosmids containing six major markers. The order of markers also agrees with the preliminary placement of a subset of probes (Wu et al. 1993), except for DXS6, which is now placed more telomeric to DXS100. This placement of DXS6 has been confirmed both by FISH and by the critical finding that DXS6 maps outside of the intervals spanned by the two deletions.

Recently the content of polymorphic markers in this region has been augmented; all are found in the contig. Because the total recombination distance in this physical interval is only $\sim 1.6 \mathrm{cM}$, the order of
Figure 2 FISH of selected YACs. (Top) Hybridization of yWXD640, containing DXS1206 (green); yWXD856, containing DXS982 (red); and cosmid CWXD715 (U234G3), containing DXS100 (green) to a male prometaphase chromosome spread. The order of markers from the centromere along Xq is DXS1206 (green), followed by DXS982 and DXS100, which are overlapping and not resolved (red/green), appearing as yellow. Inset shows an enlargement of the area containing signals. (Bottom) Hybridization of yWXD640 (DXS1206), labeled with red and green dyes, appearing as yellow; yWXD856 (DXS982), labeled with digoxigenin and detected with anti-digoxigenin-FITC (green); and CWXD715 (DXS100, red) to a male G1 PCC metaphase plate. The orientation of the chromosome from centromere to telomere is unequivocally determined by the banding pattern, and the order of the markers from the centromere along $\mathrm{Xq}$ is DXS1206 (red and green, appearing as yellow), DXS982 (green), DXS100 (red). Inset shows an enlargement of the area containing signals.

markers on the genetic map is relatively weak, and cannot be stringently compared with the physical order. In ongoing work, however, longer-range linkage and physical orders are in accord for a contig extended several megabases in both the centromeric and telomeric directions (R. Nagaraja and S. MacMillan, in prep.).

\section{Refining the Localization of the LYP Gene}

In a few cases, and notably in the case of Duchenne muscular dystrophy (see Mandel et al. 1992), interstitial deletions have localized a hereditary disease lesion to a region small enough for molecular analysis. In the case of LYP, the reduction of the target

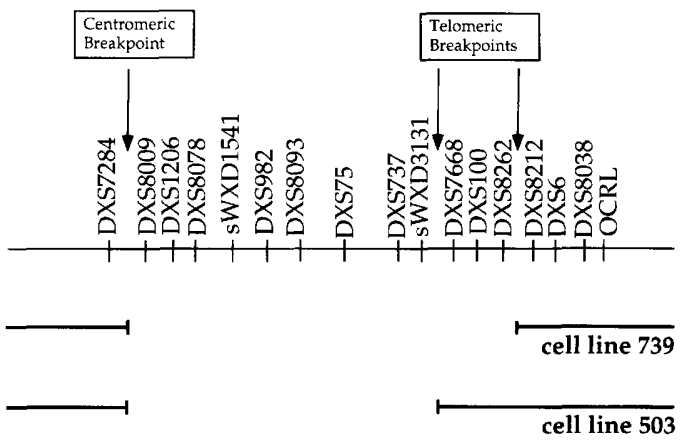

Figure 3 Localization of deletion breakpoints in DNAs from two LYP patients. For clarity, the relative locations of a small number of STSs of Fig. 1 are shown in relation to the positions of the breakpoints in the 739 and 503 deletions. The arrows indicate the location of centromeric and telomeric breakpoints. 
PORTA ET AL.

\begin{tabular}{|c|c|c|c|c|c|}
\hline Insert-end & Base pair & $\%$ GC & Insert-end & Base pair & $\% \mathrm{GC}$ \\
\hline $788 \mathrm{~L}$ & 142 & 26 & $162 \mathrm{R}$ & 155 & 45.2 \\
\hline $788 \mathrm{R}$ & 225 & 27 & $592 \mathrm{~L}$ & 189 & 34.4 \\
\hline $3163 \mathrm{~L}$ & 221 & 38 & $286 \mathrm{~L}$ & 162 & 39.6 \\
\hline $1356 \mathrm{R}$ & 126 & 20 & $839 \mathrm{R}$ & 169 & 33.8 \\
\hline $1357 \mathrm{R}$ & 127 & 19.7 & $856 \mathrm{R}$ & 198 & 27.8 \\
\hline $763 R$ & 87 & 33.4 & $5159 \mathrm{~L}$ & 164 & 29.3 \\
\hline $606 \mathrm{R}$ & 106 & 44 & $3478 \mathrm{~L}$ & 142 & 33.1 \\
\hline $606 \mathrm{~L}$ & 52 & 40.4 & $3478 \mathrm{R}$ & 151 & 43.1 \\
\hline $592 \mathrm{R}$ & 83 & 27.8 & $1829 \mathrm{~L}$ & 169 & 43.2 \\
\hline
\end{tabular}

A sample set of YAC insert-sequences across the region; all but five of them (sWXD269, 788R; sWXD1100, 162R; sWXD875; 5159L; sWXD1290, 3478R; and sWXD1291, 1829L) were too high in AT level to yield workable STSs. The overall average \% GC was 34 .

region to a $3-\mathrm{Mb}$ interval provides an entree to the next stage of the search for the gene, although this is a large target area for a single gene search. On the other hand, there are several indications that this region contains relatively few genes. First, the patients show no symptoms other than LYP, so that there are clearly no other genes in the region whose loss results in significant pathology. Second, rarecutter restriction analysis of about half the region (G. Porta, unpubl.) shows few if any of the CpG islands that are telltales for an estimated half of all genes in the genome (Antequera and Bird 1993). Third, the GC content of the region, an indicator of gene concentration (Bernardi 1993), is quite low. One indication of this is the fraction of YAC insert ends that showed high AT levels (Table 2). Also, in direct analyses of genomic DNA fractionated by gra-

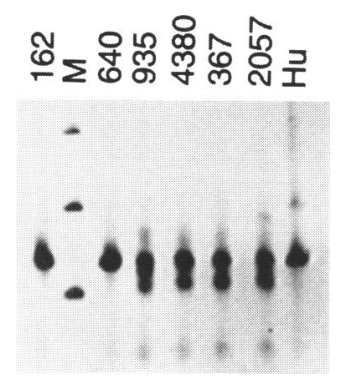

Figure 4 PCR-based amplification of STS SWXD1095 (162L) from groups of YACs at three loci in Xq25. Assays are shown for pairs of YACs from each of three loci (Fig. 1 and text): yWXD162 and 640; 935 and 4380; 367 and 2057. Lane 2 shows a set of marker DNA fragments of 50, 100, and $200 \mathrm{bp}$. The expected 61bp product appears in total human DNA (right lane) as well as in the YACs. dient centrifugation and probed with a series of markers from the region, probes for DXS100 and DXS6, for example, indicated an ambient isochore with the low GC level of $\sim 41 \%$ GC. The sum of these results suggest that the LYP gene is likely to be one of a small number encoded in the DNA that is absent in the 503 deletion.

\section{Deletion Formation and Disease Etiology}

In an increasing number of cases, starting with the classic hotspots seen in the DMD gene (Lindlof et al. 1989), recombination between very highly repetitive elements has been shown to underlie the formation of a fraction of deletions involved in human pathophysiology. For example, inter-Alu recombination has been implicated in hypercholesterolemia (Lehrman et al. 1986); hereditary C3 deficiency (Botto et al. 1992), and mucopolysaccharidosis type IVA (Hori et al. 1995). In other cases, however, local low-frequency repetitive elements have been implicated in deletion formation that leads to hereditary pathology. Two notable examples have been analyzed on the X chromosome. CRI-S232 and G1.3, which are interspersed in $\mathrm{Xp} 22.3$, have been suggested to be involved in the generation of deletions, including those that lead to Kallmann's syndrome (Ballabio et al. 1990; Li et al. 1992). Another example is the deletion formation in Xq28, which occurs in a number of cases between the multiple copies of the gene that has one of its sites in intron 22 of the factor VIII gene (Freije and Schlessinger 1992). Those deletions result in hemophilia A (Tuddenham et al. 1994).

The deletions analyzed here may provide another comparable case, because duplicated seg- 
YAC CONTIG SPANNING LYP DELETIONS IN Xo25

ments including the STS from YAC $162 \mathrm{~L}$ lie at or near the borders of the deletions. Recombination based on pairing and looping out of the homologous segments may then be a significant source of deletions resulting in LYP.

\section{METHODS}

\section{Clones and STS Content Mapping}

YACs, P1 artificial chromosomes (PACs), and STSs are referred to either by DXS numbers where available, or by their accession nos. in the Washington University Genome Center database, with the corresponding prefixes YWXD, pWXD, and sWXD (see legend to Fig. 1); details, including YAC library locations and chimera information, are in Genome Database (http://gdbwww.gdb.org) and on the Center Web page at http://genome.wustl.edu/cgm/cgm.html. YACs are available from American Type Culture Collection (ATCC). Cosmid U234G3, containing probe DXS100, was screened from the Livermore library by a PCR assay for the probe. PACs were screened from the library prepared by Dr. P. de Jong (Roswell Park Cancer Institute, Buffalo, NY). YACs yWXD7802 (yX31C9) and 7803 (yX34G7) were kindly provided by Drs. A. Coffey and D. Bentley (Sanger Centre, Hinxton, UK). Cellular DNAs with $3 \mathrm{Mb}$ (503) or $3.5 \mathrm{Mb}$ (739) deletions were as reported (Wu et al. 1993). The most probable order of markers in Figure 1 is inferred by the version 3.41 SEGMAP program of P. Green and C. Magness (University of Washington; obtained from chuck@chimera.biotech.washington.edu).

\section{FISH Method}

Cosmids and Alu-PCR amplification products from the YACs (Lengauer et al. 1992) were labeled by incorporation of biotin11-dUTP (Sigma) or digoxigenin-11-dUTP (Boehinger Mannheim) using a nick translation kit (GIBCO-BRL). Labeled DNAs in the range of $50-800 \mathrm{bp}$, with a median size of $\sim 200$ bp, were used for the in situ hybridization experiments.

In situ hybridization and washing procedures were performed as described (Srivastava et al. 1995). Triple hybridizations were done with $100 \mathrm{ng}$ each of biotin-, digoxigenin-, or biotin-digoxigenin-labeled DNA from two YACs and $50 \mathrm{ng}$ of biotin-, or digoxigenin-labeled DNA from a cosmid, plus 120 $\mu \mathrm{g}$ of human Cot 1 DNA (GIBCO-BRL), $5 \mu \mathrm{g}$ of sheared salmon sperm DNA, and $5 \mu \mathrm{g}$ of Escherichia coli tRNA in $10 \mu \mathrm{l}$ of $10 \%$ dextran sulfate, $50 \%$ formamide, and $1 \times$ SSC, and hybridized overnight at $37^{\circ} \mathrm{C}$. Biotin-labeled probes were detected using Avidin conjugated with Cy-3 (Sigma) resulting in red signal at the location of the probe on the chromosome. Digoxigeninlabeled probes was detected with antidigoxigenin conjugated with fluorescein isothiocyanate (FITC) (B.M.) and amplified with an antisheep antibody conjugated with FITC (Jackson Laboratories). This gives a green signal at the location of the probe on the chromosome.

Slides were examined under a Zeiss Axiovert fluorescent microscope. DAPI replication banding was viewed with an 02 filter set, the FITC signal with an 09 filter set, and the Cy3 signal with a 15 filter set. Images were captured using a cooled CCD (Photometrics, Tucson, AZ) and displayed on a Macintosh computer. Images were processed as described in Featherstone and Huxley (1993). Chromosomes were identified by the 4',6-diamidino-2-phenylindole (DAPI) replicational banding pattern.

\section{ACKNOWLEDGMENTS}

We are grateful to Allison Coffey and David Bentley for many useful discussions and the provision of YACs and STSs. This work was supported by grants HG00201 and HG00247, Giovanni Porta's portion was funded by the Associazione Italiana Ricerca sul Cancro, and the Telethon E 294.

The publication costs of this article were defrayed in part by payment of page charges. This article must therefore be hereby marked "advertisement" in accordance with 18 USC section 1734 solely to indicate this fact.

\section{REFERENCES}

Antequera, F. and A.A. Bird. 1993. Number of CpG islands and genes in human and mouse. Proc. Natl. Acad. Sci. 90: 11995-11999.

Ballabio, A., B. Bardoni, S. Guioli, E. Basler, and G. Camerino. 1990. Two families of low-copy number repeats are interspersed on Xp22.3: Implications for the high frequency of deletions in this region. Genomics 8: 263-270.

Bare, R.S., C.J. DeLor, K.P. Clauser, P. Hurtubise, W. Henle, J.F. Hewetson. 1974. Fatal infection mononucleosis in a family. N. Engl. J. Med. 290: 363-367.

Bernardi, G. 1993. The isochore organization of the human genome and its evolutionary history - A review. Gene 135: $57-66$.

Botto, M., K.Y. Fong, A.K. So, R. Barlow, R. Routier, B.J. Moreley, and M.J. Walport. 1992. Homozygous hereditary C3 deficiency due to a partial gene deletion. Proc. Natl. Acad. Sci. 89: 4957-4961.

Conley, M.E. 1992. Molecular approaches to analysis of $\mathrm{X}$-linked immunodeficiencies. Annu. Rev. Immunol. 10: $215-238$.

Featherstone, T. and C. Huxley. 1993. Extrachromosomal maintenance and amplification of yeast artificial chromosome DNA in mouse cells. Genomics 17: 267-278.

Freije, D. and D. Schlessinger. 1992. A 1.6-Mb contig of yeast artificial chromosomes around the human factor VIII gene reveals three regions homologous to probes for the DXS115 locus and two for the DXYS64 locus. Am. J. Hum. Genet. 51: $66-80$.

Hori, T., S. Tomatsu, Y. Nakashima, A. Uchiyama, S. Fukuda, K. Sukegawa, N. Shimozawa, Y. Suzuki, N. Kondo, T. Horiuschi, et al. 1995. Mucopolysaccharidosis type IVA: Common double deletion in the $\mathrm{N}$-acetylgalactosamine-6-sulfatase gene (GALNS). Genomics 26: $535-542$.

Kere, J., R. Nagaraja, S. Mumm, A. Ciccodicola, M. D'Urso, and D. Schlessinger. 1992. Mapping human chromosome by 
PORTA ET AL.

walking with sequence-tagged sites from fragments of yeast artificial chromosome insert. Genomics 14: 241-248.

Lehrman, M.A., D.W. Russell, J.L. Goldstein, and M.S. Brown. 1986. Exon-Alu recombination deletes 5 kilobases from the low density lipoprotein receptor gene, producing a null phenotype in familial hypercholesterolemia. Proc. Natl. Acad. Sci. 83: 3679-3683.

Lengauer, C., E.D. Green, and T. Cremer. 1992. Fluorescence in situ hybridization of YAC clones after Alu-PCR amplification. Genomics 13: 826-828.

Li, M., P.H. Yen, and L.J. Shapiro. 1992. Characterization of a low copy repetitive element $\$ 232$ involved in the generation of requent deletions of the distal short arm of the human X chromosome. Nucleic Acids Res.

20: $1117-1122$.

Lindlof, M., A. Kiuru, H. Kaariainen, H. Kalimo, H. Lang, H. Pihko, J. Rapola, H.J. Somer, M. Somer, M.L. Savontaus, et al. 1989. Gene deletions in X-linked muscular dystrophy. Am. J. Hum. Genet. 44: 496-503.

Mandel, J.L., A.P. Monaco, D. Nelson, D. Schlessinger, and H.F. Willard. 1992. Genome analysis and the human X chromosome. Science 258: 103-109.

Ogasawara, J.R., R. Watanabe-Fukunaga, M. Adachi, A. Matsuzawa, T. Kasugai, Y. Kitamura, N. Itoh, T. Suda, and S. Nagtata. 1993. Lethal effect of the anti-Fas antibody in mice. Nature 364: 806-809.

Purtilo, D.T., D. DeFlorio, L.M. Hutt, J. Bhawan, J.P.S. Yang, R. Otto, and W. Edwards. 1977. Variable phenotypic expression of an X-linked recessive lymphoproliferative syndrome. N. Engl. J. Med. 297: 1077-1081.

Seemayer, T.A., T.G. Gross, R.M. Egler, S.J. Pirruccello, J.R. Davis, C.M. Kelly, M. Okano, A. Lanyi, and J. Sumegi. 1995. $\mathrm{X}$-linked lymphoproliferative disease- twenty-five years after the discovery. Pediatr. Res. 38: 471-478.

Skare, J., B.-L. Wu, S. Madan, V. Pulijaal, D.T. Purilo, D. Haber, D. Nelson, B. Sylla, H. Grierson, and H. Nitowsky. 1993. Characterization of three overlapping deletions causing X-linked lymphoproliferative disease. Genomics 16: $254-255$.

Srivastava, A.K., T. Featherstone, K. Wein, and D. Schlessinger. 1995. YAC contigs mapping the human COL4A5 and COL4A6 genes and DXS118 within Xq21.3-q22. Genomics 26: 502-509.

Sullivan, L.J. and B.A. Woda. 1989. X-linked lymphoproliferative syndrome. Immunodeficiency Rev. 1: $325-247$.

Tuddenham, E.G., R. Schwaab, J. Seehafer, D.S. Millar, J. Gitschier, M. Higuchi, S. Bidichandani, J.M. Connor, L.W. Hoyer, A. Yoshioka, et al. 1994. Haemophilia A: Database of nucleotide substitutions, deletions, insertions, and rearrangements of the factor VIII gene, second edition. Nucleic Acids Res. 22: 3511-3533.
Wu, B-L, A. Milunsky, D. Nelson, B. Schmeckpeper, G. Porta, D. Schlessinger, and J. Skare. 1993. High-resolution mapping of probes near the X-linked lymphoproliferative disease (XLP) locus. Genomics 17: 163-170.

Wyandt, H., H. Grierson, W. Sanger, J. Skare, A. Milunsky, and D.T. Putilo. 1989. Chromosome deletion of Xq25 in an individual with $\mathrm{X}$-linked lymphoproliferative disease. $\mathrm{Am}$. J. Med. Genet. 33: $425-430$.

Zucchi, I., S. Mumm, G. Pilia, S. MacMillan, R. Reinbold, L. Susani, J. Weissenbach, and D. Schlessinger. 1996. YAC/STS map across $12 \mathrm{Mb}$ of $\mathrm{Xq} 27$ at $25 \mathrm{~kb}$ resolution, merging Xq26-qter. Genomics 34: 42-54.

Received August 12, 1996; accepted in revised form November 12, 1996.

\section{$36\lrcorner$ GENOME RESEARCH}




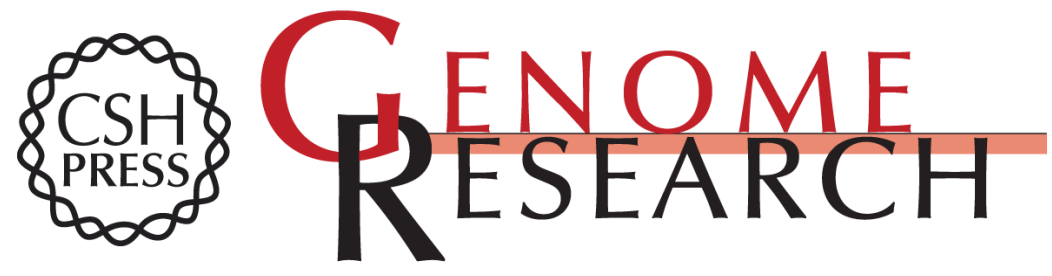

\section{5-Mb YAC STS contig at 50-kb resolution, spanning Xq25 deletions in two patients with lymphoproliferative syndrome.}

G Porta, S MacMillan, R Nagaraja, et al.

Genome Res. 1997 7: 27-36

Access the most recent version at doi:10.1101/gr.7.1.27

Supplemental Material

References

License

Email Alerting Service
http://genome.cshlp.org/content/suppl/2000/03/06/7.1.27.DC1

This article cites 25 articles, 3 of which can be accessed free at: http://genome.cshlp.org/content/7/1/27.full.html\#ref-list-1

Receive free email alerts when new articles cite this article - sign up in the box at the top right corner of the article or click here.

\section{Affordable, Accurate Sequencing.}

